\title{
Constant-Error Pseudorandomness Proofs from Hardness Require Majority
}

\author{
EMANUELE VIOLA, Northeastern University
}

\begin{abstract}
Research in the 1980s and 1990s showed how to construct a pseudorandom generator from a function that is hard to compute on more than $99 \%$ of the inputs. A more recent line of works showed, however, that if the generator has small error, then the proof of correctness cannot be implemented in subclasses of $\mathrm{TC}^{0}$, and hence the construction cannot be applied to the known hardness results. This article considers a typical class of pseudorandom generator constructions, and proves an analogous result for the case of large error.
\end{abstract}

Categories and Subject Descriptors: F.1.1 [Computation by Abstract devices]: Models of Computation

General Terms: Algorithms, Theory

Additional Key Words and Phrases: Majority, pseudorandom generator, black box, lower bound

\section{ACM Reference format:}

Emanuele Viola. 2019. Constant-Error Pseudorandomness Proofs from Hardness Require Majority. ACM Trans. Comput. Theory 11, 4, Article 19 (June 2019), 11 pages.

https://doi.org/10.1145/3322815

The construction of pseudorandom generators from hard functions is a fundamental paradigm in complexity theory. Call a function $f:\{0,1\}^{\ell} \rightarrow\{0,1\} \delta$-hard if every "small circuit" fails to compute $f$ on at least a $\delta$ fraction of the inputs. Perhaps the simplest example of the paradigm is this. Given $f$ which is $(1 / 2-\epsilon)$-hard, the repetition pseudorandom generator

$$
\begin{gathered}
P R G:\{0,1\}^{\ell \cdot s} \rightarrow\{0,1\}^{\ell \cdot s+s}, \\
P R G\left(x_{1}, x_{2}, \ldots, x_{s}\right):=\left(x_{1}, x_{2}, \ldots, x_{s}\right) \circ f\left(x_{1}\right) \circ f\left(x_{2}\right) \circ \cdots \circ f\left(x_{s}\right)
\end{gathered}
$$

has error $O(\epsilon s)$, by which we mean that small circuits cannot distinguish its output distribution from uniform with advantage more than $O(\epsilon s)$. The proof relies on the hybrid argument; see, for example, Fefferman et al. [3] and the discussion there. Hence, from hardness $1 / 2-\epsilon$ we can obtain $\Omega(1 / \epsilon)$ bits of pseudorandomness, and this is tight for black-box reductions [3].

This repetition approach has poor output/input ratio less than two. The landmark paper by Nisan [13] gave a better construction using a design $S_{1}, S_{2}, \ldots, S_{s} \subseteq\{1,2, \ldots, m\}$. Here, the generator gets an input $\sigma$ and computes from it $s$ inputs $x_{1}, x_{2}, \ldots, x_{s}$ for $f$ where $x_{i}$ is the bits of $\sigma$

Viola's work is supported by the National Science Foundation, under grant CCF-1813930.

Author's address: E. Viola, Khoury College of Computer Sciences, Northeastern University, 360 Huntington Av., \#202 WVH, Boston, MA 02115; email: viola@ccs.neu.edu.

Permission to make digital or hard copies of all or part of this work for personal or classroom use is granted without fee provided that copies are not made or distributed for profit or commercial advantage and that copies bear this notice and the full citation on the first page. Copyrights for components of this work owned by others than the author(s) must be honored. Abstracting with credit is permitted. To copy otherwise, or republish, to post on servers or to redistribute to lists, requires prior specific permission and/or a fee. Request permissions from permissions@acm.org.

(C) 2019 Copyright held by the owner/author(s). Publication rights licensed to ACM.

1942-3454/2019/06-ART19 \$15.00

https://doi.org/10.1145/3322815 
indexed by $S_{i}$.

$$
\begin{aligned}
N & :\{0,1\}^{m} \rightarrow\{0,1\}^{m+s}, \\
N(\sigma) & :=\sigma \circ f\left(x_{1}\right) \circ f\left(x_{2}\right) \circ \cdots \circ f\left(x_{s}\right) .
\end{aligned}
$$

The repetition generator can be seen as a trivial design where the $S_{i}$ are disjoint. Nisan allows us to take $s$ much larger than $m$, and so the stretch is essentially unaffected if we drop $\sigma$ from the output.

Jumping ahead, our result only applies if $\sigma$ is revealed in the output. We note that most or all applications of such generators in the literature are unaffected if $\sigma$ is revealed, while there are applications (e.g., Kinne et al. [7]) which actually require that $\sigma$ is revealed. Still, we view the case where $\sigma$ is not revealed as an interesting open problem which we discuss more in Section 3.

Starting from Less Hard $f$. If the hardness of $f$ is less, say if it is a constant bounded away from $1 / 2$, then the above approaches give only a constant number of bits of pseudorandomness. Since at least Nisan and Wigderson [14], the way around this has been to amplify the hardness of $f$ to obtain another function $f^{\prime}$ with hardness $1 / 2-\epsilon$ where $\epsilon \leq 1 / s$, and then apply a generator construction to $f^{\prime}$. The simplest and most well-known hardness amplification is the XOR lemma originating from oral presentations by Yao in the 1980s (see Goldreich et al. [4]). Here, $f^{\prime}$ is simply the XOR of $k$ independent copies of $f$ :

$$
\begin{aligned}
f^{\prime} & :\{0,1\}^{\ell \cdot k} \rightarrow\{0,1\}, \\
f^{\prime}\left(x_{1}, x_{2}, \ldots, x_{k}\right) & :=\oplus_{j \leq k} f\left(x_{j}\right) .
\end{aligned}
$$

Here, by $\oplus_{j \leq k}$ we mean the XOR over all $j \in\{1,2, \ldots, k\}$.

For a concrete example, if we combine this hardness amplification with the repetition generator, we obtain the following construction where $m=\ell k s$ :

$$
\begin{array}{rl}
G:\{0,1\}^{m} & \rightarrow\{0,1\}^{m+s}, \\
G\left(x_{i j}\right)_{i \leq s}:=\left(x_{i j}\right)_{i \leq s} & \circ \oplus_{j \leq k} f\left(x_{1 j}\right) \circ \oplus_{j \leq k} f\left(x_{2 j}\right) \circ \cdots \circ \oplus_{j \leq k} f\left(x_{s j}\right) . \\
j \leq k & j \leq k
\end{array}
$$

If we start with $f$ that has constant hardness, then in Yao's XOR lemma for any given $s$, we can take $k=O(\log s)$ to obtain hardness say $1 / 2-1 / s^{2}$, which is sufficient to show that $G$ has error $O(1 / s)$.

The extension of Nisan's generator where we first apply Yao's XOR lemma appears in the work of Nisan and Wigderson [14]. It is as follows:

$$
\begin{aligned}
N W & :\{0,1\}^{m} \rightarrow\{0,1\}^{m+s}, \\
N W(\sigma) & :=\sigma \circ \oplus_{j \leq k} f\left(x_{1 j}\right) \circ \oplus_{j \leq k} f\left(x_{2 j}\right) \circ \cdots \circ \oplus_{j \leq k} f\left(x_{s j}\right),
\end{aligned}
$$

where now all the $x_{i j}$ are computed from $\sigma$ via a design as before.

Hardness Amplification Proofs Require Majority. This hardness amplification step is problematic for restricted computational models. This is because most or all reductions that prove hardness amplification are black-box, and a line of research [1, 5, 6, 11, 17, 19, 20] has shown that these reductions cannot be implemented in a class less powerful than $\mathrm{TC}^{0}$. The sense in which this holds is made precise below, but basically the reduction circuits that amplify hardness to $1 / 2-\epsilon$ can be used to compute majority on $\Omega(1 / \epsilon)$ bits. This means that when $\epsilon$ is polynomially small, these reductions can only be used when starting from a lower bound against at least $\mathrm{TC}^{0}$, a long-standing open problem that is believed to require new techniques $[12,16]$. In particular, they cannot be used for several classes for which we do have $\delta$-hard functions for $\delta=0.1$ but not even $\delta=1 / 2-1 / \sqrt{\ell}$ 
for functions on $\ell$ bits. Such classes include $\mathrm{AC}^{0}$ circuits with parity gates $[15,18]$ or with one majority gate [2]. We refer to Grinberg et al. [5] for additional discussion and pointers.

Moreover, most proofs of pseudorandom generators $P R G:\{0,1\}^{m} \rightarrow\{0,1\}^{m+s}$ also yield error about $1 / s$. For example, in the above example of $G$ if we amplify hardness to $1 / s^{2}$, we obtain error $O(1 / s)$. And the same limitations apply to a pseudorandomness proof with error $\epsilon$ as to a hardness amplification proof to hardness $1 / 2-\epsilon[5]$.

Constant-Error Pseudorandomness. However, such limitations were not known for pseudorandomness proofs with constant error. Pseudorandom generators with constant error are important in their own right. For example, they suffice for derandomization, and are not known for several frontier classes such as $\mathrm{AC}^{0}$ with parity gates. In this article, we show that even the proofs of the constructions above with constant error require $\mathrm{TC}^{0}$. For $s$ bits of stretch, we obtain the same limitations established in Grinberg et al. [5] for amplifying hardness to $1 / 2-1 / s$. We state a more general and abstract result, where the generator computes inputs $x_{i j}$ from the seed $\sigma$ via a function $L$, then evaluates $f$ at those inputs and combines the output via a function $H$.

Let us prepare for the technical statement of the result. A black-box proof of correctness consists of a reduction showing that if the generator is broken, then the function $f$ is not hard. More formally, such a reduction is an oracle circuit $C$ that receives an input $x$ and oracle access to a distinguisher $D$ for the generator, and outputs $f(x)$ correctly on a $1-\delta$ fraction of inputs. If $D$ has small size and depth, and $C$ has small size and depth, one obtains a circuit $C^{\prime}(x)=C^{D}(x)$ also of small size and depth that computes $f$ well, thus contradicting the hardness of $f$. It is known that in this setup, the reduction circuit $C$ must use an additional advice string $\alpha$, which may arbitrarily depend on the function $f$ and the distinguisher $D$. More precisely, the reduction circuit $C$ fulfills the following guarantee: For every function $f$, and every distinguisher $D$, there exists an advice string $\alpha$ for which $\mathbb{P}_{x \in\{0,1\}^{\ell}}\left[C^{D}(x, \alpha)=f(s)\right] \geq 1-\delta$. The reader is referred to Shaltiel and Viola [17] and Grinberg et al. [5] for additional discussion. Following Viola [20] and Shaltiel and Viola [17], the theorem below shows that reduction circuits $C$ of this type can be used to distinguish uniform bits from slightly biased bits. Specifically, let us denote by $N_{1 / 2-\epsilon}^{q}$ a $q$-tuple of i.i.d. bits coming up 1 with probability $1 / 2-\epsilon$. The theorem shows how to distinguish between $N_{1 / 2}^{q}$ and $N_{1 / 2-\Omega(1 / s)}^{q}$ using roughly the same size and depth as that of the circuits $C$ (with no oracle and using the same gates as in $C$ plus gates for $H$ and $L$ ). By arguments in Shaltiel and Viola [17], we can then show how to compute majority on $\Omega(s)$ bits, and moreover the circuits $C$ must make many queries.

Theorem 0.1. There is a constant $c$ such that if $\max \{s, a, q, k\} \leq g \leq 2^{\ell / c}$ and $2^{-\ell / c} \leq \delta \leq 1 / 3$, the following holds.

Hypothesis: Let $G^{f}(\sigma)=\sigma \circ G_{1}^{f}(\sigma) \circ G_{1}^{f}(\sigma) \circ \cdots \circ G_{s}^{f}(\sigma)$ be a pseudorandom-generator construction mapping a seed $\sigma \in\{0,1\}^{m}$ and a function $f:\{0,1\}^{\ell} \rightarrow\{0,1\}$ to $m+s$ output bits defined by

$$
G_{i}^{f}(\sigma)=H\left(f\left(x_{i 1}\right), f\left(x_{i 2}\right), \ldots, f\left(x_{i k}\right)\right),
$$

where $H:\{0,1\}^{k} \rightarrow\{0,1\}$ is a function, and the $s \cdot k$ values $x_{i j}$ for $i \leq s$ and $j \leq k$ are obtained from $\sigma$ by the map $L:\{0,1\}^{m} \rightarrow\{0,1\}^{\ell \cdot s \cdot k}$. Suppose that with probability $1-1 / 1,000$ over $\sigma$, the $x_{i j}$ values $L(\sigma)$ are distinct.

Let $\left\{C^{\cdot}(., \alpha)\right\}_{\alpha \in\{0,1\}^{a}}$ be a family of $2^{a}$ oracle circuits such that for every $\alpha$ the circuit $C^{\cdot}(., \alpha)$ has size $g$, depth $\kappa$, makes $q$ oracle queries, and uses gates $\Xi \supseteq\{$ And, Or, Not $\}$.

Suppose that for every $f:\{0,1\}^{\ell} \rightarrow\{0,1\}$ and $d:\{0,1\}^{m+s} \rightarrow\{0,1\}$ such that

$$
\left|\mathbb{P}\left[d\left(G^{f}(\sigma)\right)=1\right]-\mathbb{P}\left[d\left(U_{m+s}\right)=1\right]\right| \geq \frac{1}{100},
$$


where $\sigma$ and $U_{m+s}$ are uniform in $\{0,1\}^{m}$ and $\{0,1\}^{m+s}$ respectively, there exists $\alpha \in\{0,1\}^{a}$ such that

$$
\mathbb{P}_{x \in\{0,1\}^{e}}\left[C^{d}(x, \alpha)=f(x)\right] \geq 1-\delta .
$$

Conclusion: Then for every $\epsilon=\Omega(1 / s)$ there is a circuit $t:\{0,1\}^{q} \rightarrow\{0,1\}$ such that

$$
\mathbb{P}\left[t\left(N_{1 / 2-\epsilon}^{q}\right)=1\right]-\mathbb{P}\left[t\left(N_{1 / 2}^{q}\right)=1\right] \geq 1-O(\delta),
$$

and moreover thas depth $O(\kappa)$, size $\operatorname{poly}(g, 1 / \delta)$, and only uses gates for $H$ and $L$ in addition to the gates $\Xi$.

By the arguments in Sections 5 and 6 of Shaltiel and Viola [17], the conclusion of the theorem has two corollaries:

(A) $q \geq \Omega\left(s^{2} \log (1 / \delta)\right)$. For this the fact that $t$ can be computed by small circuits is not required.

(B) There is a circuit with depth $O(\kappa)$, size poly $(g, 1 / \delta)$, and the same gates as $t$ that computes majority on inputs of length $\Omega(s)$. In particular, if $H, L, C$ are computable in $\mathrm{AC}^{0}$ or even in $\mathrm{AC}^{0}$ with parity gates, the size of $C$ must be exponential in $s^{\Omega(1)}$. By contrast, for unbounded-depth circuits the size can be polynomial. Note, for this $\delta=1 / 3$ is sufficient.

The function $L$ is the identity map in the repetition PRG, and is a projection in NW. In both cases, it is trivially computable in $\mathrm{AC}^{0}$. The function $H$ is simply parity on $k$ bits in both cases, obviously computable in $\mathrm{AC}^{0}$ with parity gates. In fact, as discussed before, in typical settings we have $k=O(\log s)$ and so this parity is even computable in $\mathrm{AC}^{0}$.

The theorem assumes that with probability $1-1 / 1,000$ over $\sigma$ the $x_{i j}$ are distinct. We now remark that for typical settings of both generators (1) and (2) this assumption holds. When each $x_{i j}$ is the projection of $\sigma$ on a set of $\ell$ bits, as long as the pairwise intersection of the sets is $\leq \ell / 2$, then the probability that two $x_{i j}$ will be equal is $\leq\left(\begin{array}{c}k s \\ 2\end{array}\right) 2^{-\ell / 2}$ by a union bound. In particular, if $k, s=\ell^{O(1)}$ the assumption is satisfied for large enough $\ell$. (The designs in Nisan [13] and Nisan and Wigderson [14] have even smaller intersection guarantees.)

The corollaries (A) and (B) are the same obtained in Grinberg et al. [5] for amplification to hardness $1 / 2-1 / s$. As explained there, the bounds are tight for hardness amplification. Specifically, the bound on the number of queries matches Klivans and Servedio [8], and the result about majority is tight because there exist proofs of Yao's XOR lemma that can be implemented in $\mathrm{AC}^{0}$ with one majority gate [9] (for a simplification of Klivans [9], due to Klivans and Vadhan, see Viola [22]). We note that such tightness extends to the generators (1) and (2), because the pseudorandomness proof from a $1 / 2-1 / s$ hard function can be implemented in $\mathrm{AC}^{0}$ with only one query [21].

Note that for constructions with small error $\epsilon$, the work Grinberg et al. [5] rules out $\mathrm{AC}^{0}$ reductions even for one bit of stretch, that is, $s=1$. In our setting of constant error $\epsilon$, we cannot do that because it is possible to get $s=$ poly $\log g$ bits of stretch via an $\mathrm{AC}^{0}$ reduction of size $g$, and our result shows that this is tight.

Theorem 0.1 would immediately follow from Grinberg et al. [5] if there was a way to turn a constant-error generator into a $1 / 2-1 / s$ hard function with a proof of correctness by a reduction that does not use majority. No such way is known, and in fact, we suspect one can derive a blackbox separation for this task along the lines of [5].

Organization. After presenting the proof, we conclude in Section 3 where we also discuss several open problems.

\section{PROOF DISCUSSION}

We explain the basic idea of the proof in the case of the repetition generator (1). Take $F$ to be a random function $F:\{0,1\}^{\ell} \rightarrow\{0,1\}$ and the distinguisher $d$ as a distribution $D$ defined as follows. Recall that the input to $D$ is a string $z$ of length $m+s$ that consists of $s k$ inputs $x_{i j}$ to $F$ and $s$ 
additional bits $b_{i}$. On input $z$, the distinguisher $D$ selects a uniform pointer $P(z) \in\{1,2, \ldots, s\}$ and then answers one if and only if the bit $b_{i}$ matches the output of the pseudorandom generator, that is, if $b_{i}=\oplus_{j \leq k} F\left(x_{i j}\right)$. Such a $D$ breaks the pseudorandom generator, as it always outputs 1 when the input is pseudorandom, but if $b_{i}$ is uniform, then the equation $b_{i}=\oplus_{j \leq k} F\left(x_{i j}\right)$ is satisfied with probability $1 / 2$.

Now imagine, for some fixed $\alpha$, a reduction circuit $C^{D}\left(x^{*}, \alpha\right)$ that is trying to compute $F\left(x^{*}\right)$ by querying $D$. Equivalently, the circuit is trying to distinguish $F_{0}:=F \mid F\left(x^{*}\right)=0$ from $F_{1}:=F \mid F\left(x^{*}\right)=1$. We can assume that in any query $z$ to $D$ the inputs $x_{i j}$ are all different (by instructing $D$ to answer 0 otherwise). Then the answers to each query in $F_{0}$ and $F_{1}$ are close: they are random bits whose statistical distance is $O(1 / s)$. When $s$ is large enough, this difference is too small to be detected by a constant-depth circuit.

Formalizing this idea presents several difficulties. First, the above definition of $D$ actually does not work. This is because, using advice, the reduction could ask queries where all the values $F\left(x_{i j}\right)$ are 0 except possibly $F\left(x^{*}\right)$. In such a situation the answers of $D$ would be always 0 under $F_{0}$, and non-zero with probability $\geq 1 / s$ under $F_{1}$. This can be detected in constant depth (by a simple Or). The fix is to pad the bits $b_{i}$ with a balanced string, so that the answer bits always come up 1 with probability bounded away from 0 and 1.

To handle advice, we use the fixed-set lemma from Grinberg et al. [5], which basically says that conditioning on a specific $\alpha$ can be thought of as fixing some values of $F$ and $D$. As in Grinberg et al. [5], this lemma appears to greatly simplify the argument.

We note that although we use this lemma from Grinberg et al. [5], and other results from Shaltiel and Viola [17], our proof presents several differences. The main one is in the choice of the oracle. In previous works, this choice is straightforward: the oracle is simply obtained by perturbing the encoding of $f$ with suitable noise, and the main focus is on the analysis. In our case, the choice is less obvious. This difference then propagates to several other parts of the proof, for example in how we handle adaptive queries.

\section{FORMAL PROOF}

Let $F:\{0,1\}^{\ell} \rightarrow\{0,1\}$ be a uniform function. We define the distinguisher $D$ as follows. Let $P$ : $\{0,1\}^{m+s} \rightarrow[3 s]$ be a uniform function. On input $z=\sigma \circ b_{1} \circ \cdots \circ b_{s} \in\{0,1\}^{m+s}$, compute

$$
\begin{array}{r}
L(\sigma)=\left\{x_{i j}\right\}_{i} \leq s \\
j \leq k
\end{array} .
$$

For simplicity we think of the output of $L(\sigma)$ as a multiset. If the $x_{i j}$ are not all distinct, then output zero. Otherwise, compute the string $v(z) \in\{0,1\}^{s}$ where the bit $i$ is the indicator of whether $b_{i}$ is the correct output of the hard function, i.e., it is the indicator of $H\left(F\left(x_{i 1}\right), F\left(x_{i 2}\right), \ldots, F\left(x_{i k}\right)\right)=b_{i}$. Return the bit $P(z)$ of the string $v(z) 0^{s} 1^{s} \in\{0,1\}^{3 s}$.

Claim 2.1. For every $F$, with probability $\Omega(1)$ over the choice of $P, D$ distinguishes the $P R G$ from uniform with advantage $\Omega(1)$.

Proof. We first prove the claim ignoring the fact that $D$ outputs 0 if the $x_{i j}$ are not all distinct.

On any input $z$ from the PRG, $D$ outputs 1 unless $P(z)$ lands on $0^{s}$. The latter happens with probability $1 / 3$ independently. Hence, with probability say $1-1 / 100$ over $P$, assuming $s$ is large enough, $D$ outputs 1 with probability $\geq 2 / 3-1 / 100$ over a uniform output of the PRG. Note, we can assume that $s$ is large enough or else the conclusion of the theorem is trivial.

We now analyze the behavior for a uniform input $u$. First note that at least say a 0.99 fraction of the strings $u \in\{0,1\}^{m+s}$ are such that $v(u)$ has Hamming weight $\leq 0.51 s$, again using that $s$ is large enough. (Note that this statement only depends on $F$, which is fixed.) For any such string $u$, 
the distinguisher outputs 1 with probability $\leq(1+0.51) / 3$ over the choice of $P(u)$. Hence, with probability $1-1 / 100$ over $P$ the distinguisher outputs 1 on at most a $(1+0.52) / 3$ fraction of those strings, and hence overall on at most a $1 / 100+(1+0.52) / 3$ fraction of the strings in $\{0,1\}^{m+s}$.

By a union bound, with probability $\geq 1-1 / 50$ over $P, D$ has an advantage of

$$
\geq(2 / 3-1 / 100)-(1 / 100+(1+0.52) / 3)=0.14 \text {. }
$$

Finally, we take into account the probability that the $x_{i j}$ are not all distinct. This happens with probability $\leq 1 / 1,000$ by assumption. Hence, we still have advantage $\geq \Omega(1)$.

By the above claim, averaging over $F$, and our assumption, there is an advice $\alpha \in\{0,1\}^{a}$ such that with probability $\geq \Omega\left(2^{-a}\right)$ over $F$ and $P$ the event $A:=" \mathbb{P}_{x \in\{0,1\}^{\ell}}\left[C^{D}(x, \alpha)=F(x)\right] \geq 1-\delta$ " happens. We now use the fixed-set lemma from Grinberg et al. [5], restated next.

Lemma 2.2. Let $N, a, q^{\prime}$ be integers. Let $Y=\left(Y_{1}, \ldots, Y_{N}\right)$ be independent random variables, each uniform over some finite set $\Sigma$. Let $A \subseteq \Sigma^{N}$ be an event such that $\mathbb{P}[Y \in A] \geq 2^{-a}$, and let $X=$ $(Y \mid Y \in A)$. For every $\eta>0$, there exists a set $B \subseteq[N]$ of size poly $\left(a, q^{\prime}, \eta^{-1}\right)$, and $\mu \in\{0,1\}^{B}$ such that for $Y^{\prime}:=\left(Y \mid Y_{B}=\mu\right)$ and $X^{\prime}:=\left(X \mid X_{B}=\mu\right)=\left(Y \mid Y_{B}=\mu, Y \in A\right)$, and every $q^{\prime}$-query decision tree $t,\left|\mathbb{P}\left[t\left(Y^{\prime}\right)=1\right]-\mathbb{P}\left[t\left(X^{\prime}\right)=1\right]\right| \leq \eta$.

The notation $X_{B}$ means the variables $X_{i}$ where $i \in B$. We apply the lemma to the $2^{\ell}+2^{m+s}$ random variables $F(x), P(z)$. We set $q^{\prime}=O(q k)$ and $\eta=\delta$.

Remark 2.3. The fixed-set lemma is only stated for variables with the same range $\Sigma$, whereas $F(x)$ and $P(z)$ have different range. However, we can, for example, rewrite $F(x)$ and $P(z)$ as fixed functions of random variables with the same range $\Sigma$ (e.g., $|\Sigma|=2 \cdot 3 s$ will do). So we can apply it in our setting as well.

The lemma gives a set $B$ of size $\operatorname{poly}\left(a, q^{\prime}, 1 / \eta\right)=\operatorname{poly}(g, 1 / \delta)$ (using $\left.a, q, k \leq g\right)$ and a string $\mu$ such that for every $x$

$$
\left|\mathbb{P}_{F, P}\left[C^{D}(x, \alpha)=F(x) \mid A,(F P)_{B}=\mu\right]-\mathbb{P}_{F, P}\left[C^{D}(x, \alpha)=F(x) \mid(F P)_{B}=\mu\right]\right| \leq \delta,
$$

where note in the second probability there is no conditioning on $A$. Here we are using that checking if $C^{D}(x, \alpha)=F(x)$ can be computed by a decision tree with access to the variables $F(x)$ and $P(z)$ making $\leq 1+q+q k=O(q k)$ queries. The tree first queries $F(x)$, then it simulates $C^{D}(x, \alpha)$, answering each oracle query $D(z)$ by querying $P(z)$ and then the $k$ corresponding values of $F$.

Recall that for every $F, P$ such that $A$ holds, we have by definition of $A$ that

$$
\mathbb{P}_{x}\left[C^{D}(x, \alpha)=F(x)\right] \geq 1-\delta .
$$

Hence, the same holds if we average over $F, P$ and further condition:

$$
\mathbb{P}_{x, F, P}\left[C^{D}(x, \alpha)=F(x) \mid A,(F P)_{B}=\mu\right] \geq 1-\delta .
$$

By Equation (3), we can drop the conditioning over $A$ and have

$$
\mathbb{P}_{x, F, P}\left[C^{D}(x, \alpha)=F(x) \mid(F P)_{B}=\mu\right] \geq 1-2 \delta .
$$

We now want to fix a "good" $x$ such that $F(x)$ is uniform and $C$ does not get too much "information" about $F(x)$ from the oracle. The first condition simply means $x \notin B$. The second is more complicated. We need to avoid that $C$ makes a query to a $z$ such that $P(z)$ is fixed to a bit that depends on $F(x)$; as that could give away the value $F(x)$ (e.g., if the answer is $F(x) \oplus 0 \oplus 0 \oplus \cdots \oplus 0$ ). For simplicity, we shall fix $F$ at all values $x \in\{0,1\}^{\ell}$ that appear in any $z \in B$. We can enlarge $B$ to include such problematic $x$ :

$$
B^{\prime}:=B \bigcup\left\{x \in\{0,1\}^{\ell}: x \in L(\sigma) \text { for some } \sigma \circ b \in B\right\} .
$$


Note that $\left|B^{\prime}\right| \leq|B|+|B| \cdot k \cdot s \leq \operatorname{poly}(g, 1 / \delta)$ (by the above bound on $|B|$ and using $k, s \leq g$ ). By averaging there exists a corresponding fixing $\mu^{\prime}$ such that again

$$
\mathbb{P}_{x, F, P}\left[C^{D}(x, \alpha)=F(x) \mid(F P)_{B^{\prime}}=\mu^{\prime}\right] \geq 1-2 \delta .
$$

Hence, we have that there exists a fixed $x^{*} \notin B^{\prime}$ such that

$$
\mathbb{P}_{F, P}\left[C^{D}\left(x^{*}, \alpha\right)=F\left(x^{*}\right) \mid(F P)_{B^{\prime}}=\mu^{\prime}\right] \geq 1-2 \delta-\left|B^{\prime}\right| / 2^{\ell} \geq 1-3 \delta,
$$

using that $\delta \geq\left|B^{\prime}\right| / 2^{\ell}$, which is implied by $2^{-\ell / c} \geq \operatorname{poly}\left(2^{\ell / c}\right) / 2^{\ell}$ because $\delta \geq 2^{-\ell / c}$ and $g \leq 2^{\ell / c}$, and hence true for a large enough $c$.

Now let us hardwire $x^{*}$ and $\alpha$ and write $C_{0}$ for $C\left(x^{*}, \alpha\right)$. So we have

$$
\mathbb{P}_{F, P}\left[C_{0}^{D}=F\left(x^{*}\right) \mid(F P)_{B^{\prime}}=\mu^{\prime}\right] \geq 1-3 \delta .
$$

Next, we produce a series of circuits $C_{i}$ to arrive to the desired $t$. Each circuit will have depth $O(\kappa)$, size poly $(g, 1 / \delta)$, will not increase the number of oracle queries, and will use the same gates $\Xi$ used by $C_{0}$, and also gates for $H$ and $L$.

Our first task is to get rid of the queries $z \in B$. If $z \in B$, then $P(z)$ is fixed. Let the corresponding evaluation be $H=H\left(F\left(x_{1}\right), F\left(x_{2}\right), \ldots, F\left(x_{k}\right)\right)$. All the $x_{i}$ belong to $B^{\prime}$, hence the corresponding values of $F$ are fixed and so $D(z)$ is fixed if $z \in B$. Construct a circuit $C_{1}$ that has all the values $D(z)$ for $z \in B$ stored in a table, and answers a query $z \in B$ with that fixed value. So we have

$$
\mathbb{P}_{F, P}\left[C_{1}{ }^{D}=F\left(x^{*}\right) \mid(F P)_{B^{\prime}}=\mu^{\prime}\right] \geq 1-3 \delta,
$$

and we now know that $C_{1}$ only makes queries $z$ where $P(z)$ is uniform. Note that because $|B|=$ $\operatorname{poly}(g, 1 / \delta)$ the size of $C_{1}$ is again poly $(g, 1 / \delta)$.

At this point, the conditioning on $P_{B^{\prime}}=\mu^{\prime}$ is immaterial, hence we drop it. Let us pause a moment to discuss the meaning of the notation used. We defined above $X_{B}$ to mean the variables $X_{i}$ where $i \in B$. Typically the number of such variables is $|B|$, but we can also use this notation when $B$ is a superset of the index set of the $X_{i}$. In this case, the number of variables $X_{B}$ may be smaller than $|B|$. This is what we mean with the notation $P_{B^{\prime}}=\mu^{\prime}$. So we can write

$$
\mathbb{P}_{F, P}\left[C_{1}^{D}=F\left(x^{*}\right) \mid F_{B^{\prime}}=\mu^{\prime}\right] \geq 1-3 \delta .
$$

Recall that $F\left(x^{*}\right)$ is still uniform conditioned on $F_{B^{\prime}}=\mu^{\prime}$ because $x^{\star} \notin B^{\prime}$. Hence, the previous equation can be rewritten as

$$
\frac{1}{2}\left(\mathbb{P}_{F, P}\left[C_{1}{ }^{D}=1 \mid F_{B^{\prime}}=\mu^{\prime}, F\left(x^{*}\right)=1\right]+\mathbb{P}_{F, P}\left[C_{1}{ }^{D}=0 \mid F_{B^{\prime}}=\mu^{\prime}, F\left(x^{*}\right)=0\right]\right) \geq 1-3 \delta .
$$

We multiply by 2 this inequality and then complement the second probability to rewrite the inequality as

$$
\mathbb{P}_{F, P}\left[C_{1}^{D}=1 \mid F_{B^{\prime}}=\mu^{\prime}, F\left(x^{*}\right)=1\right]-\mathbb{P}_{F, P}\left[C_{1}{ }^{D}=1 \mid F_{B^{\prime}}=\mu^{\prime}, F\left(x^{*}\right)=0\right] \geq 1-6 \delta .
$$

Now for $b \in\{0,1\}$ define the oracle $E_{b}$ as follows. The input is a string $w \in\{0,1, ?, 1-?\}^{s}$ with exactly one occurrence of ? (possibly as $1-$ ?). The output is obtained by replacing ? with $b$ (and $1-$ ? with $1-b)$ to obtain $w_{b} \in\{0,1\}^{s}$ and then outputting a uniformly selected bit of $w_{b} 0^{s} 1^{s}$.

Lemma 2.4. There is a distribution on circuits $C_{2}$ such that for every $b \in\{0,1\}$ we have

$$
\mathbb{P}\left[C_{2}{ }^{E_{b}}=1\right]=\mathbb{P}_{F, P}\left[C_{1}{ }^{D}=1 \mid F_{B^{\prime}}=\mu^{\prime}, F\left(x^{*}\right)=b\right] .
$$

Proof. The high-level idea is that $C_{2}$ fills the unfixed values of $F(x)$ on the fly, for every $x \neq x^{*}$, while keeping a table of its choices. More precisely, initialize the table with the values $F_{B^{\prime}}=\mu^{\prime}$. 
Then arrange the oracle gates of $C_{1}$ in levels $1,2, \ldots$ with level 1 being closest to the input. To perform the queries

$$
\begin{gathered}
z_{1}=\sigma_{1} b_{11} \cdots b_{1 s}, \\
z_{2}=\sigma_{2} b_{21} \cdots b_{2 s}, \\
\quad \cdots \\
z_{t}=\sigma_{t} b_{t 1} \cdots b_{t s}
\end{gathered}
$$

for $t \leq q$, at some level $i, C_{2}$ first computes $L^{\prime}:=L\left(\sigma_{1}\right) \cup L\left(\sigma_{2}\right) \cup \ldots \cup L\left(\sigma_{t}\right)$. For every $x \in L^{\prime}$ that is in the table, it fetches the corresponding value of $F$. For the others, except for $x^{*}$, it tosses a coin, and stores the value in the table. From these values it computes $t$ strings $w_{1}^{\prime}, w_{2}^{\prime}, \ldots, w_{t}^{\prime} \in$ $\{0,1, ?, 1-\text { ? }\}^{s}$ by computing $H$. If an evaluation of $H$ depends on the unknown value $F\left(x^{*}\right)$, then $C_{2}$ writes ? or 1-? depending on what this dependency is. (If $H$ syntactically depends on $F\left(x^{*}\right)$ but actually the other input bits of $H$ already determine the value of $H, C_{2}$ writes a value in $\{0,1\}$. This obviously never happens when $H=$ Parity.)

Recall from above that there is at most one occurrence of $x^{*}$ in each $L\left(\sigma_{i}\right)$ (or else the oracle always outputs 0 and the query is not made). Hence, each of the strings $w_{i}^{\prime}$ has $\leq 1$ occurrence of ?. Then the strings $w_{1}, w_{2}, \ldots, w_{t}$ are constructed by performing bit-by-bit equality with the $b_{i j}$. More formally, the $j$ bit of $w_{i}$ is $w_{i j}:=1 \oplus w_{i j}^{\prime} \oplus b_{i j}$, where $\oplus$ is bit-wise XOR and the expression is the indicator of the equality function (here $(1-?) \oplus 1=$ ?, etc.). For those strings $w_{i}$ that happen to be in $\{0,1\}^{s}$, the oracle query is simply answered by the circuit by returning a uniform bit of $w_{i} 0^{s} 1^{s}$. For the other strings, $C_{2}$ queries $E_{b}$.

Now we further simplify the oracle to zoom in on strings that are nearly balanced. Earlier, we had $|w|=s$; now we will have $|w|=1$. Let $E_{b}^{\prime}$ be the oracle that takes as input $w \in\{?, 1-?\}$, substitutes $b$ for ? to obtain $w_{b} \in\{0,1\}$, and then outputs a uniform bit of $w_{b} 0^{s} 1^{s}$.

Lemma 2.5. There is a distribution on circuits $C_{3}$ of size polynomial in that of $C_{2}$ and constant depth such that for every $b \in\{0,1\}$ we have

$$
\mathbb{P}\left[C_{3}^{E_{b}^{\prime}}=1\right]=\mathbb{P}\left[C_{2}^{E_{b}}=1\right] .
$$

Proof. Think of answering an oracle query to $E_{b}$ as follows. On input $w \in\{0,1, ?, 1-?\}^{s}$, write $w=w^{\prime} w^{\prime \prime}$ where $w^{\prime \prime} \in\{?, 1-?\}$. First toss a selection coin to decide if the answer will be a uniform bit from $w^{\prime}$ or from $w_{b}^{\prime \prime} 0^{s} 1^{s}$. In the former case, we can answer the query without invoking the oracle. In the latter case, the oracle query is answered using $E_{b}^{\prime}$.

Now we want to replace the oracles $E_{0}^{\prime}, E_{1}^{\prime}$ with inputs $N_{1 / 2}^{q}, N_{1 / 2-\epsilon}^{q}$. Note $E_{0}^{\prime}$ on input? outputs $N_{s /(2 s+1)}$ and on input 1-? outputs $N_{(s+1) /(2 s+1)}$. $E_{1}^{\prime}$ does the same but with ? and 1-? swapped. We use the following lemma to map pairs of distributions.

Lemma 2.6. Let $p, \gamma, \epsilon$ be such that the following quantities are in $[0,1]: 1 / 2-\epsilon, p+\gamma, p-\gamma, p+$ $\gamma+\gamma / 2 \epsilon, p+\gamma-\gamma / 2 \epsilon$. There is a distribution on functions $M:\{0,1\} \rightarrow\{0,1\}$ such that $M\left(N_{1 / 2}\right) \equiv$ $N_{p+\gamma}$ and $M\left(N_{1 / 2-\epsilon}\right) \equiv N_{p-\gamma}$, where $\equiv$ denotes equivalence as distributions.

Proof. $M(0)$ outputs 1 with probability $\alpha:=p+\gamma-\gamma / 2 \epsilon$; $M(1)$ outputs 1 with probability $\beta:=$ $p+\gamma+\gamma / 2 \epsilon$. Then

$$
\begin{aligned}
\mathbb{P}\left[M\left(N_{1 / 2}\right)\right. & =1]=(1 / 2) \beta+(1 / 2) \alpha=p+\gamma, \\
\mathbb{P}\left[M\left(N_{1 / 2-\epsilon}\right)\right. & =1]=(1 / 2-\epsilon) \beta+(1 / 2+\epsilon) \alpha=p+\gamma+\epsilon(-\beta+\alpha)=p-\gamma .
\end{aligned}
$$

Consider the circuit $C_{4}$ that on input a string $x \in\{0,1\}^{q}$ simulates $C_{3}$. Oracle query at gate $i$ on input? is answered applying Lemma 2.6 to bit $x_{i}$ of $x$ with $p=1 / 2$ and $\gamma=-1 / 2(2 s+1)$. If $x_{i}$ is 
sampled according to $N_{1 / 2}$, then we get $N_{1 / 2-1 / 2(2 s+1)}=N_{s /(2 s+1)}$, and if $x_{i}$ is sampled according to $N_{1 / 2-\epsilon}$, then we get $N_{1 / 2+1 / 2(2 s+1)}=N_{(s+1) /(2 s+1)}$, as desired. On input $1-$ ? we instead pick $\gamma=$ $+1 / 2(2 s+1)$. In both cases the hypotheses of the lemma hold for $\epsilon=\Omega(1 / s)$. This shows that

$$
\begin{aligned}
\mathbb{P}\left[C_{4}\left(N_{1 / 2}^{q}\right)=1\right] & =\mathbb{P}\left[C_{3}^{E_{0}^{\prime}}=1\right], \\
\mathbb{P}\left[C_{4}\left(N_{1 / 2-\epsilon}^{q}\right)=1\right] & =\mathbb{P}\left[C_{3}^{E_{1}^{\prime}}=1\right] .
\end{aligned}
$$

Combining this with the above lemmas and Equation (4) gives that

$$
\mathbb{P}\left[C_{4}\left(N_{1 / 2-\epsilon}^{q}\right)=1\right]-\mathbb{P}\left[C_{4}\left(N_{1 / 2}^{q}\right)=1\right] \geq 1-6 \delta .
$$

By averaging, we can fix $C_{4}$ to a circuit $t$, and this concludes the proof.

\section{DISCUSSION AND OPEN PROBLEMS}

This work points to several open problems. First, can we prove similar limitations when the seed $\sigma$ is not part of the output of the generator? Our proof strategy immediately encounters a problem because it is not clear anymore how to define the distinguisher $D$. On input $z$, which values of $F$ should $D$ use to answer? Here is a candidate definition of $D$. On input $z$, consider the string $z^{\prime}$ that is closest to $z$ in Hamming distance and that can be output by the generator. Pick a uniform index $i$ and answer $z_{i} \oplus z_{i}^{\prime}$ (suitably padded). Then we would need to understand how changing one value of $F$ affects the output of $D$. But here technical difficulties arise that we have not yet been able to overcome.

\section{Problem 1. Prove Theorem 0.1 when the seed $\sigma$ is not part of the output (for $s \gg m$ ).}

Second, what can we prove for fixed hard functions $f$ ? The result is actually false! More in detail, recall that our proof is black-box in both the use of the distinguisher $d$ and of the hard function $f$. For proofs that are only black-box in $d$ but that can be tailored to specific functions $f$ the result is false: Fefferman et al. [3] showed with an $\mathrm{AC}^{0}$ reduction that the repetition generator is pseudorandom with no error loss when starting with any "resamplable function," such as Parity. Using this, they constructed the best-known generators for classes such as $\mathrm{AC}^{0}$ with modular gates. However, it is not known how to push their techniques to Nisan-style pseudorandom generators with much better stretch. A natural proof strategy would require sampling the output distribution of the generator, which is impossible [10]. So it may be interesting to understand if the techniques in this article can be extended to handle a fixed $f$ like Parity. We remark that for this one needs a proof that further exploits that the reduction's queries are a low-complexity function of the input $x$, or a different distinguisher. This is because a reduction on input $x$ could make, for example, the query $\left(x_{1} 0 x_{2} 0 \ldots x_{t} 0\right)$ where each $x_{i}$ has the same parity as $x$. Then a distinguisher such as ours would allow one to compute the parity of $x$. And this is exactly how the proof in Fefferman et al. [3] works. Intuitively, we cannot compute such correlated $x_{i}$ for a random function $f$, and our proof formalizes the intuition.

Because Nisan's generator applied to the parity function is just the uniform distribution over a vector space, we can askthe following.

Problem 2. Understand the pseudorandomness properties of vector spaces $V \subseteq\{0,1\}^{n}$ with bitwise addition modulo 2. In particular, for how small $m$ does there exist a space of dimension $m$ that fools $A C^{0}$ with mod 3 gates? What about a single mod 3 gate? What can be proved using reductions that are black-box in the use of the distinguisher?

We remark that some of the earlier works did hold for fixed $f$. For example, Artemenko and Shaltiel proved [1] a query lower bound even for fixed $f$ (in the setting of hardness amplification with small error). 
Finally, for the NW generator (where $L$ is projection and $H$ is parity) the circuits $t$ in the conclusion of Theorem 0.1 are $\mathrm{AC}^{0}$ with parity gates. It is not clear to us how to obtain $\mathrm{AC}^{0}$ circuits (unless $k$ is small). It may be useful to think about this as a stepping stone toward handling arbitrary $H$.

\section{ACKNOWLEDGMENTS}

In a previous version, the conclusion of Theorem 0.1 had the incorrect size bound poly $(g)$ instead of poly $(g, 1 / \delta)$. Moreover, in the proof $\eta$ was set incorrectly, whereas it suffices to set $\eta=\delta$. We thank a referee for pointing this out. The change in the size bound from $\operatorname{poly}(g)$ to $\operatorname{poly}(g, 1 / \delta)$ does not affect the corollaries mentioned after Theorem 0.1 , because there either $\delta$ is a constant or the size bound is irrelevant. Another referee, whom we thank as well, pointed out that in the conclusion of Theorem 0.1 we can take $t$ to be a fixed circuit, as opposed to a distribution over circuits as also stated in a previous version. We are also grateful to the referees for several other detailed comments which improved the presentation.

\section{REFERENCES}

[1] Sergei Artemenko and Ronen Shaltiel. 2014. Lower bounds on the query complexity of non-uniform and adaptive reductions showing hardness amplification. Computational Complexity 23, 1 (2014), 43-83. DOI : http://dx.doi.org/10. 1007/s00037-012-0056-2

[2] James Aspnes, Richard Beigel, Merrick Furst, and Steven Rudich. 1994. The expressive power of voting polynomials. Combinatorica. A fournal on Combinatorics and the Theory of Computing 14, 2 (1994), 135-148.

[3] Bill Fefferman, Ronen Shaltiel, Christopher Umans, and Emanuele Viola. 2013. On beating the hybrid argument. Theory of Computing 9 (2013), 809-843.

[4] Oded Goldreich, Noam Nisan, and Avi Wigderson. 1995. On Yao's XOR Lemma. Technical Report TR95-050. Electronic Colloquium on Computational Complexity. www.eccc.uni-trier.de/.

[5] Aryeh Grinberg, Ronen Shaltiel, and Emanuele Viola. 2018. Indistinguishability by adaptive procedures with advice, and lower bounds on hardness amplification proofs. In IEEE Symposium on Foundations of Computer Science (FOCS'18). Available at http://www.ccs.neu.edu/home/viola/.

[6] Dan Gutfreund and Guy Rothblum. 2008. The complexity of local list decoding. In 12th International Workshop on Randomization and Computation (RANDOM'08).

[7] Jeff Kinne, Dieter van Melkebeek, and Ronen Shaltiel. 2012. Pseudorandom generators, typically-correct derandomization, and circuit lower bounds. Computational Complexity 21, 1 (2012), 3-61. DOI: http://dx.doi.org/10.1007/ s00037-011-0019-z

[8] Adam Klivans and Rocco A. Servedio. 2003. Boosting and hard-core sets. Machine Learning 53, 3 (2003), $217-238$.

[9] Adam R. Klivans. 2001. On the derandomization of constant depth circuits. In Workshop on Randomization and Computation (RANDOM'01). Springer.

[10] Shachar Lovett and Emanuele Viola. 2012. Bounded-depth circuits cannot sample good codes. Computational Complexity 21, 2 (2012), 245-266.

[11] Chi-Jen Lu, Shi-Chun Tsai, and Hsin-Lung Wu. 2011. Complexity of hard-core set proofs. Computational Complexity 20, 1 (2011), 145-171. DOI : http://dx.doi.org/10.1007/s00037-011-0003-7

[12] Moni Naor and Omer Reingold. 2004. Number-theoretic constructions of efficient pseudo-random functions. Fournal of the ACM 51, 2 (2004), 231-262.

[13] Noam Nisan. 1991. Pseudorandom bits for constant depth circuits. Combinatorica. A fournal on Combinatorics and the Theory of Computing 11, 1 (1991), 63-70.

[14] Noam Nisan and Avi Wigderson. 1994. Hardness vs randomness. fournal of Computer and System Sciences 49, 2 (1994), 149-167.

[15] Alexander Razborov. 1987. Lower bounds on the dimension of schemes of bounded depth in a complete basis containing the logical addition function. Akademiya Nauk SSSR. Matematicheskie Zametki 41, 4 (1987), 598-607. English translation in Mathematical Notes of the Academy of Sciences of the USSR, 41, 4 (1987), 333-338.

[16] Alexander Razborov and Steven Rudich. 1997. Natural proofs. fournal of Computer and System Sciences 55, 1 (Aug. 1997), 24-35.

[17] Ronen Shaltiel and Emanuele Viola. 2010. Hardness amplification proofs require majority. SIAM fournal on Computing 39, 7 (2010), 3122-3154.

[18] Roman Smolensky. 1987. Algebraic methods in the theory of lower bounds for Boolean circuit complexity. In 19th ACM Symposium on the Theory of Computing (STOC'87). ACM, 77-82. 
[19] Emanuele Viola. 2004. The complexity of constructing pseudorandom generators from hard functions. Computational Complexity 13, 3-4 (2004), 147-188.

[20] Emanuele Viola. 2006. The Complexity of Hardness Amplification and Derandomization. Ph.D. Dissertation. Harvard University.

[21] Emanuele Viola. 2007. Pseudorandom bits for constant-depth circuits with few arbitrary symmetric gates. SIAM fournal on Computing 36, 5 (2007), 1387-1403.

[22] Emanuele Viola. 2009. On the power of small-depth computation. Foundations and Trends in Theoretical Computer Science 5, 1 (2009), 1-72.

Received July 2018; revised February 2019; accepted March 2019 